This page is intentionally left blank 


\section{C++ PROGRAMMMING}
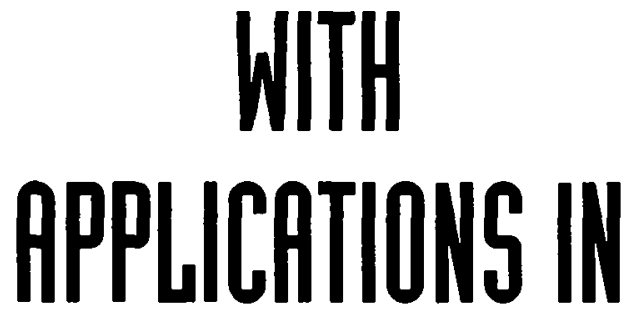

Administration, Finance and Statistics

\section{Willi-Hans Steeb \& Fritz Solms}

Rand Afrikaans University, Johannesburg, South Africa 


\section{Published by}

World Scientific Publishing Co. Pte. Ltd.

P O Box 128, Farrer Road, Singapore 912805

USA office: Suite 1B, 1060 Main Street. River Edge, NJ 07661

UK office: 57 Shelton Street, Covent Garden, London WC2H 9HE

\section{British Library Cataloguing-in-Publication Data}

A catalogue record for this book is available from the British Library.

\section{$\mathrm{C}^{++}$PROgramming With applications in ADMINISTRATION, FINANCE AND STATISTICS}

Copyright $@ 2000$ by World Scientific Publishing Co. Pte. Ltd.

All rights reserved. This book, or parts thereof, may not be reproduced in any form or by any means, electronic or mechanical, including photocopying, recording or any information storage and retrieval system now known or to be invented, without written permission from the Publisher.

For photocopying of material in this volume, please pay a copying fee through the Copyright Clearance Center, Inc., 222 Rosewood Drive, Danvers, MA 01923, USA. In this case permission to photocopy is not required from the publisher.

ISBN $981-02-4066-X$

This book is printed on acid and chlorine free paper.

Printed in Singapore by Fulsland Offset Printing 


\section{Preface}

$\mathrm{C}++$ is the most widely available and used object-oriented programming language. It is a very useful language that is successfully utilized by many programmers in many application areas. It is a reasonably carefully thought-out language where the design is based partly on acknowledged principles and partly on solid experience and feedback from actual use. The language is much more complicated and complex than, say, C and PASCAL. The key concepts of data abstraction and object-oriented programming are new to most people. One can use $\mathrm{C}++$ effectively as a more strongly type checked $\mathrm{C}$ with a small amount of simple data abstraction and library use. This programming language has all the tools to write compact and comprehensive programs for finance, administration and statistics. Many of the most experienced individuals and organizations in the industry and academia use $\mathrm{C}++$. Many programmers strongly prefer $\mathrm{C}++$ over procedural languages such as $\mathrm{C}$ and PASCAL and deem the feature set provided by $\mathrm{C}++$ as close to essential for their applications. $\mathrm{C}++$ supports object-oriented programming. It supports it pretty well for real life applications. Its type system and general model of the world are coherent and relevant to real applications. $\mathrm{C}++$ and its various implementations have a solid core where different compilers tend to agree and where the code generated is reliably good.

This book provides software solutions to problems in finance, administration and statistics. Most standard methods used in these fields are given together with their programs in $\mathrm{C}++$. In some cases the output of the programs is also displayed. Moreover the book gives an introduction to $\mathrm{C}++$. Most of the programs are written in $\mathrm{C}++$. Some of the programs are written in C. All the standard methods such as number sorting, name sorting, applications of function templates, applications of class templates, inheritance, virtual classes, string manipulations are included.

In chapter 2 we give some basic programs in $\mathrm{C}++$. These programs are very helpful for the beginners. They are also the building blocks for more complex applications. Chapter 3 is devoted to the manipulation of strings. The class concept is introduced in chapter 4. Chapter 5 and 6 deal with function and class templates. A collection of introductory programs are given. Sorting and searching algorithms are implemented in $\mathrm{C}++$ in chapter 7 . Chapter 8 gives a collection of useful classes. They include string classes, vector classes, a matrix class, linked lists, trees and a date class. Chapter 9 is devoted to output/input streams. Here we consider $\mathrm{C}$ and $\mathrm{C}++$ programs. In chapter 10 a collection of applications in finance are given. Applications in administration are considered in chapter 11. Problems in statistics are considered in chapter 12. Chapter 13 describes exception handling. The standard template library is introduced in chapter 14 and several examples are given. 
The level of presentation is such that one can study the subject early on in ones education in science. There is a balance between practical programming and the underlying language. The book is ideally suited for use in lectures on $\mathrm{C}, \mathrm{C}++$ and object-oriented programming. The beginner will also benefit from the book. Almost all $\mathrm{C}++$ programs in the book comply to the ANSI $\mathrm{C}++$ standard.

The reference list gives a collection of text books useful in the study of the computer language $\mathrm{C}++$. There are a number of good text books for $\mathrm{C}++$ available [3], [6], [7], [8], [11], [13], [9]. We also refer to The Annotated $C++$ Reference Manual by Margret Ellis and Bjarne Stroustrup [6]. For data structures we refer to Budd [4]. For applications in science we refer to Tan Kiat Shi and W.-H. Steeb [14] and Steeb [12]. The standard template library is described by Schildt [10], Ammeraal [1] and Budd [5].

For a comprehensive discussion of the Boyer-Moore algorithm, the Floyd-Warshall algorithm, Dijkstra algorithm, the sorting methods and the divide-and-conquer strategy we refer to Baase (1989) [2].

Without doubt, this book can be extended. If you have comments or suggestions, we would be pleased to have them. The email address of the first author is:

whs@na.rau.ac.za

The web page of the first author is:

http://zeus.rau.ac.za/

One of the authors (W.-H. Steeb) thanks his students from the Computational Science Programme of the National University of Singapore for stimulating discussions on object-oriented programming. They are: Fong Wei Leng, Heng Kok Liang, Tan Kiat Shi, Lee Wan Sie, Gan Chee Kwan, Toh Che Chou, Koh Mei Leng, Lee Yih, And Mieng Hwang, Teo Wee Chung, Lee Choon Meng. 


\section{Keywords in $\mathrm{C}++$}

C ++ has the following keywords:

Table: $\mathrm{C}++$ language keywords

\begin{tabular}{llll}
\hline asm & double & new & switch \\
auto & else & operator & template \\
break & enum & private & this \\
case & extern & protected & throw \\
catch & float & public & try \\
char & for & register & typedef \\
class & friend & return & union \\
const & goto & short & unsigned \\
continue & if & signed & virtual \\
default & inline & sizeof & void \\
delete & int & static & volatile \\
do & long & struct & while \\
\hline
\end{tabular}

Remark. asm is reserved for implementors to include a machine specific facility to pass information to an assembler. extern indicates that external linkage is to be made to this item. Obviously the placement of such an item must be known at compile time and hence must be implicitly static. 


\section{Basic Data Types of $\mathrm{C}++$}

The basic data types or primitive data types are as follows:

\begin{tabular}{|l|l|l|}
\hline Type & May also be specified as & Comment \\
\hline char & & Holds a character \\
\hline unsigned char & & Unsigned version of char \\
\hline signed char & & Signed version of char \\
\hline int & $\begin{array}{l}\text { signed int } \\
\text { signed }\end{array}$ & $\begin{array}{l}\text { A machine word that holds an } \\
\text { integer number }\end{array}$ \\
\hline unsigned int & unsigned & Unsigned version of int \\
\hline short & $\begin{array}{l}\text { signed short int } \\
\text { signed short }\end{array}$ & May have less precision than an int \\
\hline unsigned short & unsigned short int & Unsigned version of short \\
\hline long & long int & May have more precision than an int \\
\hline unsigned long & unsigned long int & Unsigned version of long \\
\hline long int & signed long int & \\
\hline float & & A number held in floating point form \\
\hline double & & May have more precision than a float \\
\hline long double & & $\begin{array}{l}\text { May have more precision than a } \\
\text { double }\end{array}$ \\
\hline
\end{tabular}

Note: A float, double and long double are stored as a floating point number. The rest of the basic data types are stored as an exact quantity.

The unsigned types will be able to store a larger positive number than their signed types.

The precision of items declared with these data types may vary, depending on the compiler or machine used.

The representation of char may be either signed or unsigned. This is to allow implementors the choice of the architectural representation of a char.

As can be seen from the table above, there is no basic data type for boolean values. $\mathrm{C}++$ like $\mathrm{C}$ before, uses integer values to represent true and false. Newer $\mathrm{C}++$ compilers have a boolean data type called bool.

\begin{tabular}{|c|c|c|}
\hline Truth value & $\mathbf{C}++$ delivers & $\mathbf{C}++$ takes a truth value \\
\hline true & 1 & Any value other than 0 \\
\hline false & 0 & 0 \\
\hline
\end{tabular}




\section{$\mathrm{C}++$ Operators}

The following precedence table shows a summary of $\mathrm{C}++$ operators in order of decreasing precedence. Each box holds operators of the same level of precedence.

For example

int $u, x, y, z$;

$\mathrm{x}=3 ; \mathrm{y}=9 ; \quad \mathrm{z}=2$;

$u=x+y \% z$;

firstly evaluates the remainder of $y \% z$ and then adds this to $x$ and assigns the result to $u$. Another example is

*g.value $\quad(* g)$.value

The member selection operator . has higher precedence then the dereferencing operator $*$. Hence $* g$. value dereferences $g$. value, whereas $(* g)$. value dereferences $g$ and then selects the member value of the object to which $g$ points. The expression $(* g)$. value can also be written as $g \rightarrow$ value. The expression

int $r=m \% n / p ;$

first evaluates $m \% n$ then we divide and finally the assignment is done. Thus the right hand side evaluates from left to right.

The use of the optrators given in following table will be illustrated with sample programs throughout this text. In $\mathrm{C}++$ one can define operators for ones own data types. For example if one defines a data type for rational numbers, then one can define the addition, subtraction, multiplication etc. for this data type. This facility is called operator overloading. All operators can be overloaded (see discussion on operator overloading later in the text) except for

When overloading operators, one should bear in mind that the order of precedence and the syntax remains the same as that for the basic data types. 


\begin{tabular}{|c|c|c|}
\hline Operator & operator name & example \\
\hline$::$ & scope resolution & class_name: : member \\
\hline$::$ & global & : name \\
\hline++ & preincrement & ++ lvalue \\
\hline-- & predecrement & -- lvalue \\
\hline . & member selection & object. name \\
\hline$\rightarrow$ & member selection & pointer $>$ member \\
\hline [] & array subscription & pointer $[$ expr $]$ \\
\hline() & function call & $\operatorname{expr}\left(\operatorname{expr} r_{-}\right.$list $)$ \\
\hline () & value construction & type (expr_list) \\
\hline sizeof & size of object & sizeof expr \\
\hline sizeof & size of type & sizeof (type) \\
\hline$=$ & one's complement & expr \\
\hline$!$ & not & !expr \\
\hline,+- & unary plus and minus & -expr and +expr \\
\hline & address of & \&lvalue \\
\hline * & dereferencing & $* \operatorname{expr}$ \\
\hline new & create (allocate memory) & new type \\
\hline delete & deallocate pointer memory & delete pointer \\
\hline delete [] & free array memory & delete[] pointer \\
\hline () & cast (type conversion) & (type) expr \\
\hline.$*$ & member section & object.*pointer-to-member \\
\hline$->*$ & member section & pointer $>*$ pointer-to-member \\
\hline$*, 1, \%$ & multiply, divide, modulo (remainder) & expr / expr \\
\hline,+- & add, subtract & expr + expr \\
\hline$\langle<\rangle\rangle$, & shift left , shift right & expr $\ll<$ expr \\
\hline$\langle,\langle=\rangle\rangle=$, & relational operators & expr $<$ expr \\
\hline$\pi=$ & equal & $\operatorname{expr}==\operatorname{expr}$ \\
\hline$!=$ & not equal & expr $!=\operatorname{expr}$ \\
\hline t & bitwise AND & expr \& expr \\
\hline- & bitwise exclusive OR & expr ${ }^{\circ} \operatorname{expr}$ \\
\hline 1 & bitwise inclusive OR & expr I expr \\
\hline 28 & logical AND & expr et expr \\
\hline 11 & logical inclusive OR & expr 11 expr \\
\hline$?:$ & conditional expression & expr ? expr : expr \\
\hline$=$ & simple assignment & lvalue $=\operatorname{expr}$ \\
\hline$*=, /=,+=,-=$ & multiply and assignment, $\ldots$ & lvalue $*=$ expr \\
\hline$<<=,>>=$ & shift left (right) and assign & l"alue $<<=$ expr \\
\hline$z=, \mid=, n=$ & AND (OR, XOR) and assignment & เvalue $\mathrm{expr}$ \\
\hline throw & throw exception & throw expr \\
\hline , & comma (sequencing) & expr, expr \\
\hline++ & postfix increment & lvaluet+ \\
\hline-- & postfix decrement & lvalue-- \\
\hline
\end{tabular}




\section{Contents}

1 Introduction 1

1.1 Aim of Object-Oriented Programming . . . . . . . . . . . 1

1.1 .1 Information Hiding . . . . . . . . . . . . . . . 2

1.1 .2 Inheritance . . . . . . . . . . . . . . . . . . 2

1.1 .3 Polymorphism . . . . . . . . . . . . . . . . . . 3

1.1 .4 Templates . . . . . . . . . . . . . . . . . . . 3

1.1.5 Standard Template Library . . . . . . . . . . . . . . . 3

1.2 Why $\mathrm{C}++$. . . . . . . . . . . . . . . 4

$2 \mathrm{C}++$ Basics 5

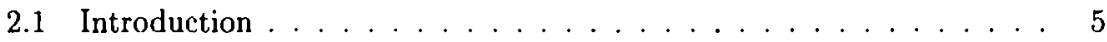

2.2 A Simple C++ Program . . . . . . . . . . . . 7

2.3 Basic Data Types . . . . . . . . . . . . . . . . . . . . . 9

2.4 Arithmetic Operations . . . . . . . . . . . . 11

2.5 ASCII Table and Types Conversion . . . . . . . . . . . . 14

2.6 Precedence Table . . . . . . . . . . . . . . . . . . . . . 16

2.7 Pointers and References . . . . . . . . . . . . . . . 18

2.8 Control Statements . . . . . . . . . . . . . . . . . . . . 21

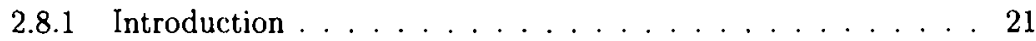

2.8 .2 The if Statement . . . . . . . . . . . . . . 22

2.8.3 The for Loop, while Loop, do-while Loop . . . . . . . . . . . . 24

2.8 .4 The switch Statement . . . . . . . . . . . . 26

2.9 Arrays . . . . . . . . . . . . . . . . . . . . . 28



2.11 The sizeof Operator . . . . . . . . . . . . . . . . . . 37

2.12 The new and delete Operators . . . . . . . . . . . 38

2.13 Arrays of Pointers . . . . . . . . . . . . . . . . . . . 42

2.14 Logical AND, Logical OR and Logical NOT . . . . . . . . . . 46

2.15 Pass by Value, Pass by Reference . . . . . . . . . . . . . 48

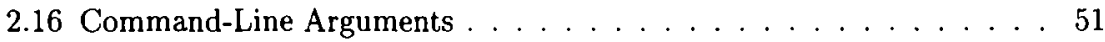

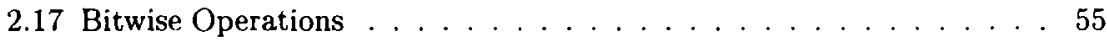

2.18 Shift Operations . . . . . . . . . . . . . . . . 57

2.19 Passing a Function to a Function . . . . . . . . . . 59

2.20 Recursion . . . . . . . . . . . . . . . . . 60

2.21 Inline Assembly Language $\ldots \ldots \ldots \ldots \ldots \ldots$ 
2.22 Time Header File . . . . . . . . . . . . . . . . . . . . . 64

2.23 Random Numbers . . . . . . . . . . . . . . . . . . 66

2.24 Constants and Inline Functions $\ldots \ldots \ldots$. . . . . . . . 67

2.25 Jump Statements . . . . . . . . . . . . . . . . . . . . 69

2.26 The static Keyword . . . . . . . . . . . . . . . 71

2.27 Structures . . . . . . . . . . . . . . . . . 72

3 String Manipulations $\quad \mathbf{7 5}$

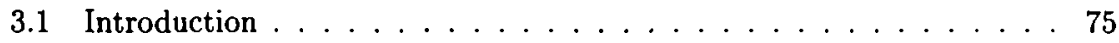

3.2 Digit Conversion to Character and String . . . . . . . . . . 76

3.3 Character in String . . . . . . . . . . . . . . 77

3.4 Append a Character to a String . . . . . . . . . . . . 78

3.5 Searching for a Character in a Given String . . . . . . . . 79

3.6 Delete a Character from a String . . . . . . . . . . . . . 80

3.7 Replacement of a Character by a Character . . . . . . . . . . 81

3.8 Swapping Characters in a String . . . . . . . . . . 82

3.9 First $n$ Characters from a String . . . . . . . . . . . 83

3.10 Substring in String . . . . . . . . . . . . . . 84

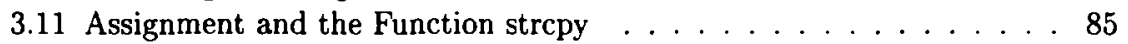

3.12 Permutations of a Word . . . . . . . . . . . . . 86

3.13 String within String . . . . . . . . . . . . . . . . . . 88

3.14 Comparing Strings $\ldots \ldots \ldots \ldots$

3.15 Length of a String . . . . . . . . . . . . . . . 92

3.16 Permutations of Characters in a String . . . . . . . . . 93

3.17 Swapping Parts of a String . . . . . . . . . . . . . . 95

3.18 String Handling using Recursion . . . . . . . . . . . . . . . 96

3.19 Concatenating Strings _. . . . . . . . . . . . . . . . . . 98

3.20 Replacing a Substring in a String . . . . . . . . . . 100

3.21 Reversing the Characters in a String . . . . . . . . . . . 102

3.22 Boyer and Moore's Algorithm . . . . . . . . . . . . . . . . . 104

3.23 Subsets of a Given Set of Strings . . . . . . . . . . . . . 107

3.24 Counting Characters and Digits in a Phrase . . . . . . . . . 108

3.25 Counting Characters and Words in a Phrase . . . . . . . . . 109

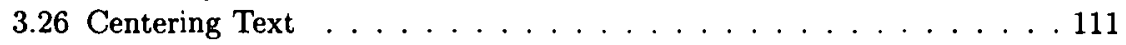

3.27 The Function getline $\ldots \ldots \ldots . \ldots \ldots$

4 The Class Concept 113

4.1 Introduction . . . . . . . . . . . . . . . . . 113

4.2 What is a Class ? . . . . . . . . . . . . . . . . 115

4.3 Constructors and Destructors . . . . . . . . . . . . . 116

4.4 Copy Constructor . . . . . . . . . . . . . . . . . . . . . 119

4.5 Operator Overloading . . . . . . . . . . . . . . . . . . . . 120

4.6 The Keyword this . . . . . . . . . . . . . . . . . . . . 121

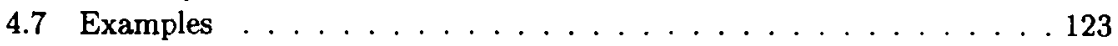

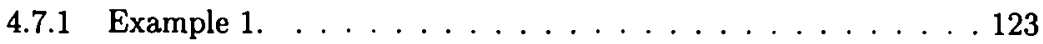




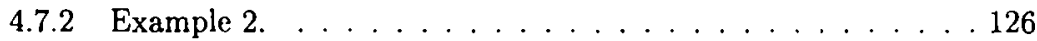

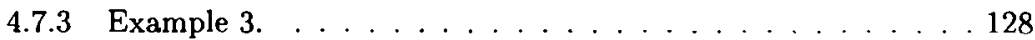

4.7.4 Example 4. . . . . . . . . . . . . . . . . . . . 130

4.7.5 Example 5. . . . . . . . . . . . . . . . . 133

4.8 Friend . . . . . . . . . . . . . . . . . . . . 135

4.9 Derivation and Inheritance . . . . . . . . . . . . . . . 137

4.10 Virtual Functions . . . . . . . . . . . . . . . . 140

5 Function Templates 143

5.1 Introduction . . . . . . . . . . . . . . . . . 143

5.2 Examples . . . . . . . . . . . . . . . . . . 144

6 Class Templates $\quad 151$

6.1 Introduction . . . . . . . . . . . . . . . . 151

6.2 Examples . . . . . . . . . . . . . . . . . . . . 152

6.2 .1 A Template Vector Class . . . . . . . . . . . . . . . . 152

6.2 .2 A Template Stack Class . . . . . . . . . . . . . . . 156

6.2.3 A Template Stack Class with Node . . . . . . . . . . . 158

6.2 .4 Two Generic Data Types . . . . . . . . . . . . . . . . . 162

7 Sorting and Searching $\quad 163$

7.1 Introduction . . . . . . . . . . . . . . . . . 163

7.2 Largest Number in an Array . . . . . . . . . . . . . . . . . . 164

7.3 Quicksort and Bubblesort . . . . . . . . . . . . 165

7.4 Shellsort . . . . . . . . . . . . . . . . . . . . 167

7.5 Using the Function bsearch $\ldots \ldots \ldots \ldots \ldots \ldots$

7.6 Using the Function lsearch . . . . . . . . . . . . . . . . 172

7.7 Name Sorting . . . . . . . . . . . . . . . . . . . . . . . 173

7.8 Name and Number Sorting Using Templates . . . . . . . . . . . 177

7.9 Student Names and Student Numbers . . . . . . . . . . . . . . . 181

7.10 Search for Names in a List . . . . . . . . . . . . . . . . . . . 183

7.11 Names of Employees in a Table . . . . . . . . . . . . . 184

7.12 The Divide-and-Conquer Methodology . . . . . . . . . . . . 186

7.13 Sorting Vectors . . . . . . . . . . . . . . . 187

8 Useful Classes 189

8.1 Introduction . . . . . . . . . . . . . . . . . . . 189

8.2 String Class . . . . . . . . . . . . . . . . . . . 190

8.2 .1 Introduction . . . . . . . . . . . . . . . . . . 190

8.2 .2 A Simple String Class . . . . . . . . . . . . . . . . 191

8.2 .3 Advanced String Classes . . . . . . . . . . . . . . . . 194

8.2 .4 Standard Library and String Class . . . . . . . . . . 200

8.3 Vector Class . . . . . . . . . . . . . . . . . . . . . . . . . . 204

8.3.1 Introduction . . . . . . . . . . . . . . . . . 204

8.3.2 A Simple Vector Class . . . . . . . . . . . . . . 205 
8.3.3 Construction of an Advanced Vector Class . . . . . . . . . . 209

8.3.4 Listing of Vector Class . . . . . . . . . . . . . . . . 217

8.4 Matrix Class . . . . . . . . . . . . . . . . . . . . 2225

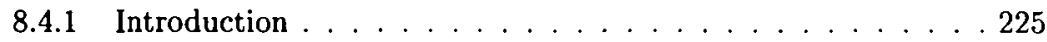

8.4 .2 Listing of Matrix Class . . . . . . . . . . . . . . . . 228

8.5 Linked Lists . . . . . . . . . . . . . . . . . . . . . . . . . . . . 237

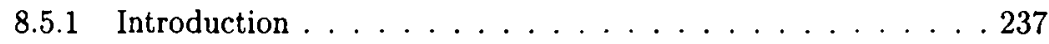

8.5 .2 Simple Linked Lists . . . . . . . . . . . . . . . . . . . . . . . . . . . . . .

8.5.3 Singly Linked Circular List . . . . . . . . . . . . . . . 243

8.5 .4 Recursive List . . . . . . . . . . . . . . . . . . . 245

8.5.5 Linked List and Templates . . . . . . . . . . . . . . . . 248

8.5.6 Linked List and Iterators . . . . . . . . . . . . . . . . . . . . . . . . . . . . . .

8.5.7 Intrusive List . . . . . . . . . . . . . . . . . . . 258

8.5.8 Generalized Lists . . . . . . . . . . . . . . . . . . . 263

8.6 Binary Trees . . . . . . . . . . . . . . . . . . . . . 269

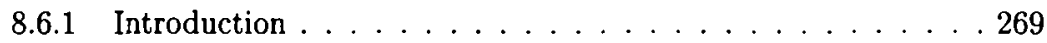

8.6.2 A Simple Binary Tree . . . . . . . . . . . . . . . 270

8.6.3 Binary Trees and Templates . . . . . . . . . . . . . 272

8.7 A Set Class . . . . . . . . . . . . . . . . . . . . . . 276

8.8 A Date and Month Class . . . . . . . . . . . . . . . . . . . . 282

8.8.1 Introduction . . . . . . . . . . . . . . 282

8.8.2 Listing of Complete Month and Date Classes . . . . . . . 296

9 File Manipulations $\quad 305$

9.1 Introduction . . . . . . . . . . . . . . . 305

9.2 C Style File Manipulations . . . . . . . . . . . . . . . . . . . 308

9.2 .1 Output to File . . . . . . . . . . . . . . . . . . 308

9.2.2 Reading String from File . . . . . . . . . . . . . . . . . . . . 309

9.2 .3 Word Count in File . . . . . . . . . . . . . . . . . . . . . . . . . . . . . . . .

9.2.4 File Searching Program . . . . . . . . . . . . . . . 311

9.2.5 Counting Characters in a File . . . . . . . . . . . . . 313

9.2.6 Class and Standard Output . . . . . . . . . . . . . . 316

9.2.7 The Functions fseek, fread, fwrite . . . . . . . . . 318

9.2.8 The Function fscanf . . . . . . . . . . . . . . 321

$9.3 \mathrm{C}++$ Style File Manipulation $\ldots \ldots \ldots 323$

9.3 .1 Output to File . . . . . . . . . . . . . . . 323

9.3.2 Reading String from File . . . . . . . . . . . . . . . 324

9.3.3 String Replacement in File . . . . . . . . . . . . . 325

9.3.4 Reading and Writing of File . . . . . . . . . . . 327

9.3.5 Writing File to Another File . . . . . . . . . . . . 331

9.4 Binary File Operations . . . . . . . . . . . . . . . . . 333

9.5 The Functions seekp and seekg $\ldots \ldots \ldots \ldots \ldots 34$

9.6 The Function isatty . . . . . . . . . . . . . 335

9.7 The Function getenv $\ldots \ldots \ldots \ldots \ldots \ldots \ldots$ 
10 Applications in Finance $\quad 337$

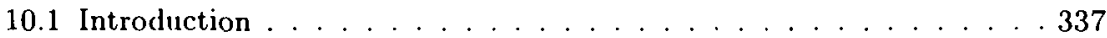

10.2 Payroll . . . . . . . . . . . . . . . . . . . . . . . . . . . . . .

10.3 Loan and Interest . . . . . . . . . . . . . . . . . . . . . . . . . . . .

10.4 Amortization . . . . . . . . . . . . . . . . . . . . . . . . . . .

10.5 Calculating the Loan Period . . . . . . . . . . . . . . . 346

10.6 Periodic Savings . . . . . . . . . . . . . . . . . . . . . . . . . 348

10.7 Compound Amount . . . . . . . . . . . . . . . . . . . . 352

10.8 The Floyd-Warshall Algorithm. . . . . . . . . . . . . . 356

10.9 The Dijkstra Algorithm . . . . . . . . . . . . . . . 359

10.10Linear Programming and Simplex Method . . . . . . . . 362

11 Applications in Administration $\quad 375$

11.1 Introduction . . . . . . . . . . . . . . . . . . . . . .

11.2 Names, Address and Inheritance . . . . . . . . . . . . . 376

11.3 Line Editor and Nested Classes . . . . . . . . . . . . . . . . . . 379

11.4 Mergesort Applied to a List . . . . . . . . . . . . . . . . 386

11.5 Linked List of Strings . . . . . . . . . . . . . . . . . . 390

11.6 Data Encryption . . . . . . . . . . . . . . . . . . . . 394

11.7 Vector Class and a Dictionary . . . . . . . . . . . . . . . 397

11.8 Spelling Checker . . . . . . . . . . . . . . . . . . . 399

11.9 Huffman Code . . . . . . . . . . . . . . . . . . . 403

11.10 Eliza . . . . . . . . . . . . . . . . . . . 409

11.11 Counting the Letters in a Phrase . . . . . . . . . . . 414

11.12The Farmer, Wolf, Goat and Cabbage Problem . . . . . . . . 420

11.13Roman Numbers . . . . . . . . . . . . . . . . . . . . . . . 427

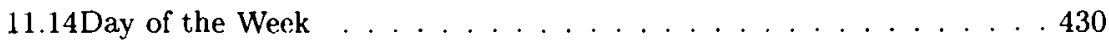

11.15 Gregorian Calender . . . . . . . . . . . . . . 431

11.16 Records . . . . . . . . . . . . . . . . . 436

11.17Finite State Machine . . . . . . . . . . . . . . . . . 443

11.18Realtor . . . . . . . . . . . . . . . . 446

12 Applications in Statistics 453

12.1 Introduction . . . . . . . . . . . . . . . . 453

12.2 Arithmetic, Harmonic and Geometric Mcan . . . . . . . . 454

12.3 Mean Value and the Standard Deviation . . . . . . . . . 458

12.4 Histogram for Series of Numbers . . . . . . . . . . . . . . 461

12.5 Data Fitting by Least Squares . . . . . . . . . . . . . . . 463

12.6 Curve Fitting . . . . . . . . . . . . . . . . . . 467

12.7 Pseudo-Random Number Generator . . . . . . . . . . . . . . 473

12.8 Correlation Coefficient . . . . . . . . . . . . . . 475

12.9 Autocorrelation Function . . . . . . . . . . . . . . . . 4777

12.10Random Number Generator and $\pi \ldots \ldots$. . . . . . . 480 
13 Exception Handling $\quad 485$

13.1 Introduction . . . . . . . . . . . . . . . . 485

13.2 Error States . . . . . . . . . . . . . . . . . . . . 485

13.3 Exception Handling . . . . . . . . . . . . . . . . 486

14 The Standard Template Library 503

14.1 Introduction . . . . . . . . . . . . . . . . . 503

14.2 The Namespace Concept $\ldots \ldots \ldots \ldots \ldots \ldots 50 \ldots \ldots$

14.3 The Vector Class . . . . . . . . . . . . . . . . . 506

14.4 The List Class . . . . . . . . . . . . . . . . . . . . 510

14.5 The Stack Class . . . . . . . . . . . . . . . 512

14.6 The Queue Class . . . . . . . . . . . . . . . . . . . . . 514

14.7 The Deque Class . . . . . . . . . . . . . . . . 516

14.8 The Bit Set Class . . . . . . . . . . . . . . . . . 518

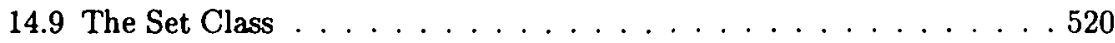

14.10The Map Class . . . . . . . . . . . . . . . . . . . . 522

14.11The Algorithm Class . . . . . . . . . . . . . . . . 525

Bibliography $\quad \mathbf{5 2 7}$

$\begin{array}{ll}\text { Index } & \mathbf{5 2 9}\end{array}$ 